\title{
EL MONTAJE DE LA VÍA DE ALTA VELOCIDAD
}

\section{(THE CONSTRUCTION OF THE HIGH SPEED TRACK)}

Ángel Higueras Martínez. Ingeniero de Caminos

Jefe de Superestructura AVE

\section{RESUMEN}

El artículo pretende pasar somera revista a los elementos que constituyen la superestructura, su distribución y montaje en obra, asi como las medidas de la vía para proceder a su recepción y puesta en servicio a las velocidades para las que fue proyectada.

\section{SUMMARY}

This article attempts to give a brief summary of the elements which make up the superstructure, their distribution and erection at the work site, and the measurements of the track, through to its reception and start-up at the speed for which it was designed.

\section{INTRODUCCIÓN}

La línea Madrid-Sevilla, con una longitud total de 471 $\mathrm{km}$, es la primera línea española que, además de tener características de Alta Velocidad, tiene ancho de vía internacional.

Esta circunstancia ha influido notablemente en la construcción de su superestructura, fundamentalmente por dos motivos.

En primer lugar, los materiales son de características distintas a las del resto de los empleados en la red ferroviaria española y ha habido que fabricarlos en un plazo de tiempo récord, adaptando las fábricas a las nuevas tecnologías necesarias para estos nuevos materiales, sin contar con que, en casos como el de los desvíos, ha sido necesario un esfuerzo adicional en la concepción de nuevos diseños.
En segundo lugar, el transporte de los materiales desde las fábricas a la obra ha tenido que hacerse utilizando primero las vías de la red con ancho RENFE y después ser distribuidos a lo largo de una línea de ancho distinto, que estaba dividida en numerosos tajos de obra, habida cuenta del corto espacio de tiempo previsto para su construcción. Ello ha requerido de un complejo plan logístico que ha hecho necesaria la instalación de 9 intercambiadores de ejes, además de un parque de acopio en Alcolea.

Por otra parte, a la logística del transporte de materiales hasta la obra, ha habido que añadir la logística propia de su distribución en cada tajo, dado que la ocupación de vía ha habido que compartirla con la maquinaria instaladora del tendido aéreo y de los cables de telecomunicaciones, operaciones realizadas en los mismos plazos que el montaje de vía en la mayoría de los tajos. 
Por todo ello no es exagerado considerar que la nueva línea de alta velocidad Madrid-Sevilla marca un hito histórico en la construcción ferroviaria española, no sólo por el tipo de tráfico a que se dedica, sino también por el gran reto que ha supuesto bajo todos los aspectos.

Se describen los trabajos realizados en la colocación de vía, poniendo especial atención tanto en aquellas actividades realizadas en función de la dificultad de la propia obra, como en aquellas tareas y procedimientos característicos de la condición de Alta Velocidad de la línea.

Por último, es de destacar que la colocación de vía se inició el 2 de octubre de 1989, estando terminada en julio de 1991, salvo tramos puntuales de unión en Madrid-Atocha y Sevilla-Sta. Justa. Los rendimientos medios obtenidos en el tendido de vía, sin nivelar, han sido entre 6 y $8 \mathrm{~km}$ de vía doble al mes. En algunos casos el rendimiento ha llegado a alcanzar el valor de 10,5 $\mathrm{km}$ al mes. Etos rendimientos han dependido fundamentalmente del ritmo de llegada al tajo de los trenes carrileros.

\section{LOS MATERIALES}

Se considera que la superestructura de una línea de ferrocarril la constituyen los materiales que se encuentran situados encima de la capa de forma, y que son:

- El subbalasto y el balasto.

- Las traviesas.

- Los carriles.

- Los aparatos de vía.

Las características del subbalasto y del balasto para poder ser empleados en una línea de Alta Velocidad están definidas en las Normas RENFE de Vía y en la ficha UIC-719. El espesor total de estas capas está en función de una serie de parámetros relacionados con el tipo de línea de que se trate. En la línea Madrid-Sevilla este espesor mínimo es de $55 \mathrm{~cm}$. Fijado el espesor de balasto bajo traviesa en $30 \mathrm{~cm}$, el espesor restante corresponde al subbalasto.

El subbalasto es una capa interpuesta entre la platafor- ma y el balasto, constituida por una grava bien graduada que contiene como mínimo un $30 \%$ de árido procedente de machaqueo. El grado de compactación a obtener es, como mínimo, del $103 \%$ de la densidad máxima obtenida del ensayo Próctor Normal y su capacidad portante, medida con placa de carga, debe ser de $1.200 \mathrm{~kg} / \mathrm{cm}^{2}$.

Sus misiones son:

- Protección de la parte superior de la plataforma contra la erosión y el hielo.

- Evacuación de las aguas superficiales ya que, por sus características de impermeabilidad, se estima que el $80 \%$ de las mismas se evacuan por su superficie.

- Colaborar en el reparto de las cargas.

- Evitar la contaminación del balasto con el material de las capas inferiores.

Sobre el subbalasto se coloca una primera capa de balasto, operación que se ha realizado de dos maneras distintas, según el tajo de la obra:

- Con extendedora. Este procedimiento garantiza, además de una colocación uniforme del material con el espesor prefijado, la homogeneidad de la composición granulométrica en toda la capa de balasto, lo que es decisivo para la posterior calidad del asiento de la vía.

Con este sistema se pueden colocar en una primera capa $24 \mathrm{~cm}$ de balasto, lo que supone además de un mayor volumen inicial (menor ocupación de vía posterior para transporte y descarga del mismo) un menor número de levantes y nivelaciones de vía.

- Extendiendo el balasto con motoniveladora y compactándolo mediante rodillos. En este caso el espesor de balasto bajo traviesa estará comprendido entre 15 y $18 \mathrm{~cm}$, por lo que será necesario, como mínimo, hacer un bateo y nivelación para alcanzar el espesor que se consigue al utilizar la extendedora.

En ambos casos y para evitar daños en las traviesas, hay que rebajar el balasto en la zona central de las mismas, para conseguir que apoyen solamente en la zona de carga.

Esta primera capa de balasto se transporta desde la cantera a la obra en camiones. El resto del balasto, a colocar durante el proceso de montaje de vía, se reparte con trenes de tolvas cautivos en la línea. 
Las traviesas son de hormigón monobloque, pudiendo ser pretensadas o postensadas. La sujeción empleada es la HM elástica.

Se han producido en 5 fábricas repartidas por la geografía española y se han transportado a la línea trenes que, dotados de ejes intercambiables, permitían acceder a la línea. Estos trenes con una composición de 9 plataformas MMQ transportaban 160 traviesas cada una, lo que supone un total de 1.440 traviesas por tren completo.

Respecto al carril, en una vía éste es el elemento sustentador del material rodante, actuando como dispositivo para su guiado, constituyendo, por tanto, el elemento principal de la vía. Cualquier irregularidad en el plano de la superficie de rodadura provoca esfuerzos dinámicos supletorios que actúan sobre ésta, creando defectos geométricos o ampliando los existentes, lo que se traduce, en definitiva, en un mayor mantenimiento. Asimismo, estas irregularidades producen en los vehículos solicitaciones anormales que afectan al confort del viajero.

Estos problemas se agravan con el aumento de velocidad, habiendo sido necesario establecer nuevos criterios de rectitud de carril, tanto en el plano vertical como en el horizontal, así como las tolerancias admisibles para los carriles a utilizar en líneas con velocidades de explotación superiores a los $200 \mathrm{~km} / \mathrm{h}$, toda vez que los criterios contenidos en la ficha UIC-860 resultaban insuficientes para estas velocidades.

El enderezado del carril, en los procesos de fabricación de colada continua, se efectúa por medio de enderezadores de rodillos, lo que provoca en el mismo ciertas irregularidades en los planos vertical y horizontal.

La onda que se produce en el carril por rodillos enderezadores (longitudes de onda entre 0,3 a más de $3 \mathrm{~m}$ ) tiene su importancia cuando se estudian bajo un carácter temporal, es decir, la frecuencia con la que aparece al paso de las circulaciones, ya que puede entrar en resonancia con las frecuencias propias de las masas suspendidas y no suspendidas de los vehículos. Las variables que definen la frecuencia de aparición de la onda son la velocidad de circulación y la longitud de onda del carril, ya que la longitud de onda que más perturba la estabilidad en la marcha de los vehículos es aquella que corresponde al perímetro de los rodillos enderazadores (ORE D-148 Rapport 7).

No obstante, el factor determinante en la fabricación del carril es aquel que lo hace económicamente realiza- ble. Actualmente, se admite, de forma general, que para longitudes de onda superiores a $1,5 \mathrm{~m}$, amplitudes de $0,3 \mathrm{~mm}$ representan la calidad máxima que se puede obtener.

El carril utilizado en la línea Madrid-Sevilla es de 60,34 $\mathrm{kg} / \mathrm{m}$, de calidad 90 (UIC) resistente al desgaste, habiéndose fabricado en colada continua y barras elementales de $36 \mathrm{~m}$. Se ha transportado al parque de soldadura de REDALSA (Valladolid) donde se forman barras largas de $288 \mathrm{~m}$, mediante la unión de 8 barras elementales, por soldadura eléctrica de contacto, lo que permite reducir el número de soldaduras aluminotécnicas a efectuar en obra.

El carril, ya en barras largas soldadas (BLS) de $288 \mathrm{~m}$, se ha transportado a obra en trenes carrileros por la red de F.C. RENFE. Estos trenes transportaban 30 BLS y tenían una longitud total de 384 metros.

Otros elementos complementarios en una vía férrea han sido los Aparatos de Dilatación, que se sitúan en aquellos puentes y viaductos que, por sus características, pueden llegar a adquirir deformaciones de cierta magnitud.

En un puente la vía está ligada, de una forma casi fija, a la obra de fábrica, por lo que el aparato de dilatación ha de compensar las variaciones de longitud y movimientos del puente respecto a la vía existente.

El puente se mueve, en primer lugar, como consecuencia de las variaciones de temperatura, retracción y fluencia y, en segundo lugar, por las fuerzas de frenado y arranque de los vehículos, movimientos que se transmiten al carril sobre el balasto, produciendo tracciones y compresiones que se añaden a las propias debidas a las variaciones de temperatura.

El conjunto de estas tensiones no debe sobrepasar el máximo admitido, $72 \mathrm{~N} / \mathrm{mm}^{2}$ en compresión y $92 \mathrm{~N} / \mathrm{mm}^{2}$ en tracción. En los puntos en los cuales la tensión en el carril supere estos valores se requiere la instalación de aparatos de dilatación.

En esta línea se han colocado Aparatos de Dilatación sólo en los viaductos hiperestáticos. Las carreras utilizadas han sido de 340,500 y $830 \mathrm{~mm}$.

Finalmente hay que referirse a los Aparatos de Vía los que por su importancia, tanto de diseño como por la problemática específica, se tratan en un artículo independiente (DESVÍOS). 


\section{LA DISTRIBUCIÓN DE LOS MATERIALES EN OBRA}

El problema inicial que hubo que resolver para realizar el suministro de carriles y traviesas para Alta Velocidad se derivaba del distinto ancho de vía con que se construía el NAFA con relación al ancho RENFE del resto de la Red. Lógicamente, los carriles y traviesas deben llegar cargados sobre vagón hasta el mismo tajo de montaje de vía, por lo que en primer término había que resolver este problema.

Se estudiaron inicialmente distintas soluciones, que en síntesis eran: montar la vía con traviesa polivalente y ancho RENFE, efectuando el cambio de ancho al final; montar una vía auxiliar de ancho RENFE a lo largo de todo el trazado, con un acceso por tramo, que se utilizaría para descarga de materiales, y luego renovar dicha vía desde el final del tramo hacia el principio; construir parques de acopio al principio de cada tramo, desde donde se cargarían los materiales a trenes de ancho internacional cautivos en obra; o diseñar una instalación suficientemente fiable y operativa que permitiera cambiar ejes a los trenes carrileros y de traviesas, sin necesidad de descargarlos.

De las opciones descritas, la primera no se estimó aconsejable para un ferrocarril de alta velocidad, con unas traviesas no suficientemente experimentadas. La segunda opción era inviable, dada la longitud de la vía auxiliar necesaria, casi $500 \mathrm{~km}$, al no existir suficiente material de segundo uso para su construcción, al margen del sobrecoste que este tendido implicaría.

La tercera alternativa era viable, pero tenía el inconveniente del coste de construcción de todos los parques de acopio precisos, de la duplicación forzosa de medios de transporte (composiciones de ancho RENFE para el transporte hasta el parque y de ancho internacional en cada tramo para transporte al tajo de montaje), así co. mo el sobrecosto originado por la descarga y carga intermedias, unido al tiempo necesario para realizar estas operaciones.

Evidentemente, la cuarta alternativa era la mejor si se diseñaba la instalación adecuada que permitiera realizar el cambio de ejes en un plazo razonable, pues su efecto en caso de aplicarse equivalía a construir el nuevo tra- zado con el mismo ancho de vía que el resto de la Red, desde el punto de vista de la logística de materiales.

Esencialmente, la instalación diseñada para la operación de cambio de ejes consta de una nave de $16 \times 40 \mathrm{~m}$, en cuyo interior, y a lo largo de toda su longitud, se halla un foso longitudinal de $4,5 \mathrm{~m}$ de ancho y $0,85 \mathrm{~m}$ de profundidad, sobre el cual se monta vía estuchada de ancho RENFE y ancho internacional. En el centro de la nave, $y$ en sentido perpendicular al anterior, existe otro foso de 1,5 m de ancho y $15 \mathrm{~m}$ de longitud, a lo largo del cual se desplaza una mesa con regulación de altura, que es la que sirve para bajar los ejes de un ancho montados en el tren que accede al intercambiador, y aportar los ejes de ancho distinto con los que saldrá la composición del intercambiador.

La operación de cambiar los 36 ejes de un tren de traviesas dura entre dos y tres horas, empleándose entre 4 y 5 para cambiar los 68 ejes de un tren tipo Robel de transporte de carril.

La mayor dificultad con la que se ha encontrado la logística de materiales para esta obra se deriva del hecho de que se ha realizado sin stocks en los tajos de obra, por lo que ha sido preciso coordinar perfectamente la circulación y llegada de trenes a los intercambiadores con objeto de no provocar paralizaciones en el montaje de vía.

Los trenes carrileros transportaban habitualmente $30 \mathrm{ba}$ rras de carril de $288 \mathrm{~m}$, lo que supone un total de 8.640 metros de carril por tren. Los de traviesas transportaban usualmente un total de 1.440 piezas cada uno, por lo que por cada tren carrilero se precisaban cinco trenes de traviesas.

Para asegurar el movimiento de trenes dentro de los tajos de obra, se han adaptado a ancho internacional un total de 24 locomotoras, y 93 tolvas tipo TT 270.000 de $36 \mathrm{~m}^{3}$ de capacidad para el transporte y descarga de balasto en vía.

Por último, el montaje de la nueva línea ha exigido dotar a cada tajo de montaje de vías auxiliares y desvíos que permitieran por un lado la descarga de los trenes carrileros en obra, y por otro la maniobrabilidad de los trenes y maquinaria de vía dentro de los tramos de vía ya montados. 


\section{EL MONTAJE DE VÍA}

\section{Replanteo de la vía}

El replanteo constituye la primera operación a realizar para el montaje de la vía, y debe llevarse a cabo con antelación para poder hacerlo de acuerdo con los datos teóricos.

La importancia fundamental que tiene el replanteo en las circulaciones ferroviarias, donde los vehículos siguen el trazado de la vía sin posibilidad de modificar su trayectoria, hace que deba ser riguroso, puesto que con la maquinaria de vía, aunque corrige los defectos relativos, no los elimina totalmente.

Por otra parte, hay que considerar que el paso a una opción de explotación con velocidades de $250 \mathrm{~km} / \mathrm{h}$ y superiores, supera lo que hasta ahora se ha considerado como el límite de la explotación ferroviaria convencional, para entrar de lleno en una nueva cultura ferroviaria de Alta Velocidad.

Si bien en lo referente a infraestructura ferroviaria existía una experiencia en España que, complementada con las recomendaciones recogida en las fichas UIC, permitía establecer una metodología de ejecución que garantizara el cumplimiento de los criterios de calidad fijados, en lo referente a superestructura no existía la misma experiencia.

Tradicionalmente, el método empleado para replantear una vía se basaba en un estaquillado y paqueteado de la misma para, aplicando el método de Chappellet sobre regularización de curvas por corrección de sus flechas, llegar al trazado definitivo, ya que toda curva imaginable posee un diagrama de flechas que le es propio.

Los sistemas de detección y corrección de defectos, instalados en la maquinaria de vía, tanto en el plano horizontal (alineación) como en el vertical (nivelación) se basan en la medida de flechas tomando como referencia la propia máquina.

Estos sistemas actúan como un filtro, restituyendo de distinta manera los diversos componentes de la señal de entrada de acuerdo con su longitud de onda. A esta relación entre las señales de entrada y las obtenidas se la conoce como función de Transferencia del Sistema de Medida.

Este sistema puede utilizarse para medir defectos de longitudes de onda inferiores a 2 veces la longitud de la cuerda, pero no tiene validez para defectos de longitud de onda mayores.

Dado que la mayor cuerda utilizable en el momento actual es de $20 \mathrm{~m}$, se podrían medir y corregir defectos de longitudes de onda inferiores a 40 metros.

Por otra parte, las longitudes de onda de los defectos geométricos que afectan a los vehículos ferroviarios aumentan al aumentar la velocidad. Las bajas frecuencias que pueden afectar a las masas suspendidas están en el orden de 1 a $2 \mathrm{~Hz}$, que corresponden a longitudes de onda espaciales de los defectos geométricos, que pueden llegar hasta los $80 \mathrm{~m}$ para velocidades del orden de $300 \mathrm{~km} / \mathrm{h}$, lo que obliga, al aumentar la velocidad, a detectar y corregir defectos de longitud de onda crecientes.

De lo anterior se deduce que los sistemas tradicionales de medida y corrección, basados en el método de las flechas, que se han venido utilizando con éxito en las líneas convencionales, no son operativos cuando la velocidad es superior a los $200 \mathrm{~km} / \mathrm{h}$, ya que las cuerdas a emplear plantean grandes problemas constructivos.

Por ello fue necesario establecer un sistema de replanteo que permitiera garantizar la posición absoluta de la vía en una íntima relación con la electrificación, y garantizase a la vez poder reproducir en todo momento su posición.

Replantear una vía con este sistema presenta una serie de ventajas, entre las que se pueden citar:

- Tener la vía siempre en la misma posición que se montó, evitando los desplazamientos laterales y, por tanto, las tensiones longitudinales que se producen en los carriles por esta causa.

- Permite mantener mejor la posición relativa de la vía y la catenaria.

- Utilizar cuerdas de mayor longitud permite reducir los errores de medida y, a la vez, las ondulaciones de la vía alrededor de su eje teórico, disminuyendo los excesos de aceleración lateral.

- En los casos de paso obligado con gálibo estricto, facilita el mantenimiento del mismo. 


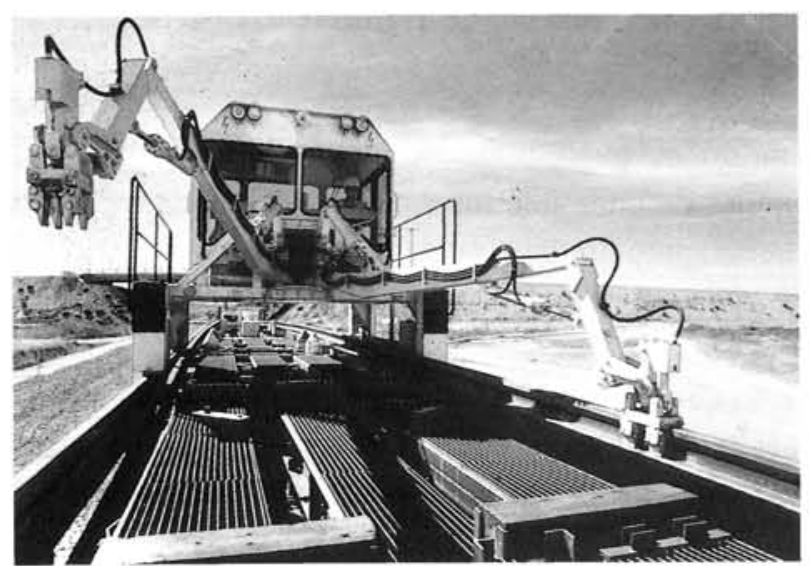

Pórtico de descarga de carriles.

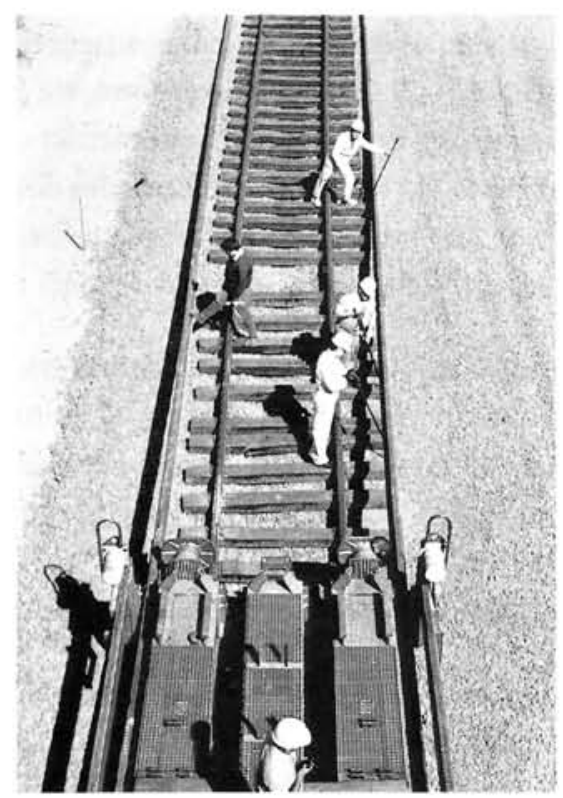

Descarga de carril sobre via auxiliar.

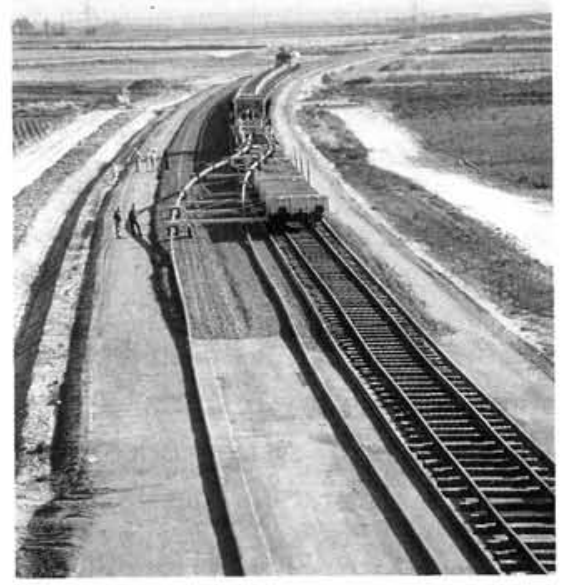

(c) Consejo Superior de Pescarga de carril Rara fíáa paralela. Licencia Creative Commons 3.0 España (by-nc)
Un replanteo con estas características permitirá, en una conservación futura, poder reproducir, en cualquier momento, los datos iniciales de trazado, con las ventajas de todo tipo que ello conlleva.

\section{Organización del montaje de vía}

Una vez extendida la primera capa de balasto el montaje de vía se realiza en diversas fases que, con ligeras variantes en alguno de los tramos de obra, son las siguientes:

\section{a) Colocación de vía auxiliar}

El proceso de colocación de vía se inicia con el montaje de la vía auxiliar. Se utiliza carril usado de 45 ó $54 \mathrm{~kg} / \mathrm{m}$ $\mathrm{y}$ traviesas de madera procedentes de levante. Al proceder de ancho RENFE $(1.668 \mathrm{~mm})$ se da la vuelta a las traviesas y se fijan por ese lado los carriles en Ancho Internacional $(1.435 \mathrm{~mm})$. Las traviesas se suelen poner a $1 \mathrm{~m}$, o más, de separación para aligerar el peso de la vía auxiliar haciéndola más manejable.

\section{b) Descarga del tren carrilero}

La descarga la realiza el mismo tren utilizando las 3 plataformas auxiliares que lleva y que disponen de "brazos" articulados instalados en una grúa móvil, que se desplaza a lo largo de toda la longitud del tren.

Los carriles se descargan -teniendo en cuenta el lado activo definido al efectuar las soldaduras eléctricas en taller-sobre la vía auxiliar en su lugar de empleo, una barra en el exterior y otra en el interior para la vía directora. Las barras correspondientes a la vía paralela se pueden descargar, bien en su situación de montaje o en el entre eje, según se disponga o no de dispositivos de guiado y separadores.

Una vez descargadas las barras, son separadas y alineadas para poder ser utilizadas como camino de rodadura de pórticos. Esta operación se realiza con especial cuidado para no producir deformaciones en el carril.

\section{c) Embridado y posicionado de barras largas al ancho de pórticos}

Esta operación se realiza en la vía directora con objeto de que los pórticos de trabajo utilicen como rodadura la vía formada provisonalmente por los carriles nuevos, directamente apoyados sobre el balasto. 
Esta vía se prolonga con unos carriles auxiliares en una longitud de unos $200 \mathrm{~m}$. Estos carriles son de 45 ó 54 $\mathrm{kg} / \mathrm{m}$, por lo que es necesario utilizar cupones mixtos como elemento de transición con el carril nuevo de 60 $\mathrm{kg} / \mathrm{m}$. La longitud de $200 \mathrm{~m}$ viene determinada por la de un tren de traviesas de 9 plataformas MMQ, con 1.440 traviesas, necesarias para montar 3 barras de 288 metros.

\section{d) Levante, transporte y tendido de vía auxiliar}

Utilizando los pórticos se va levantando la vía auxiliar por tramos para ir posicionando la vía nueva en su lugar. Los pórticos depositan la vía auxiliar, por parejas, sobre carros o "diplorys" que la transportan, mediante una "dresina", hasta el extremo opuesto en el sentido del avance de la colocación de vía. Allí son descargadas mediante una retro o grúa, $\mathrm{u}$ otro sistema, para ir tendiendo y embridando, dando así continuidad a la vía auxiliar.

\section{e) Perfilado-cajeado del balasto}

Levantada la vía auxiliar se perfila y cajea el balasto utilizando una pareja de pórticos. Con este cajeado se asegura el apoyo correcto de las traviesas en sus extremos.

\section{f) Descarga de traviesas}

Utilizando una segunda pareja de pórticos se van descargando traviesas. Los pórticos toman las traviesas del tren correspondiente situado en la zona de carriles auxiliares. La descarga de las traviesas de la vía directora se hace en dos fases de $30+30$ traviesas que se realizan sucesivamente rodando por la vía formada por las BLS de $288 \mathrm{~m}$ anteriormente descargadas. Las traviesas, al descargar, quedan ya a $60 \mathrm{~cm}$ de distancia procediéndose después a los mínimos movimientos con barras.

Las de la vía paralela se descargan por medio de grúas móviles, con un sistema similar de descarga, dejándolas separadas también cada $60 \mathrm{~cm}$.

\section{g) Posicionado y sujeción de carril}

Una vez descargadas las traviesas se procede a la colocación de los carriles sobre los apoyos de las traviesas, con posicionadoras de carril, sin dañar los carriles, las traviesas, ni los elementos de sujeción de las mismas. (c) Consejo Superior de Investigaciones Cientíicas Licencia Creative Commons 3.0 España (by-nc)

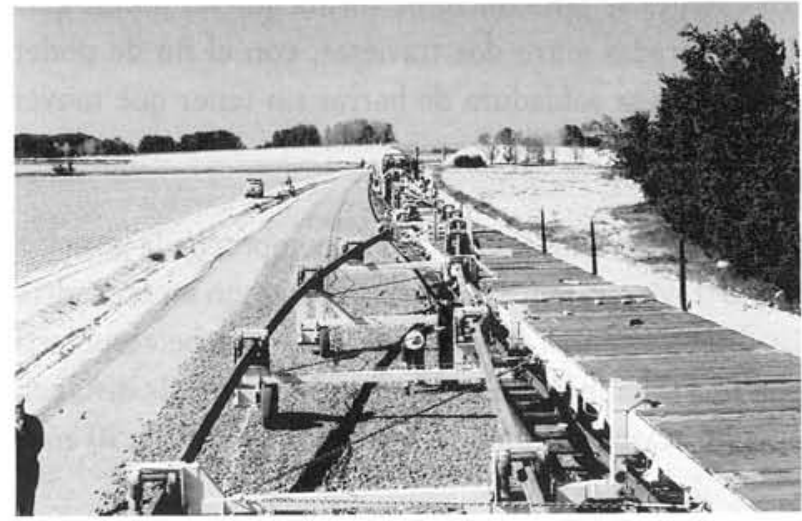

Separador de carril para descarga en vía paralela.

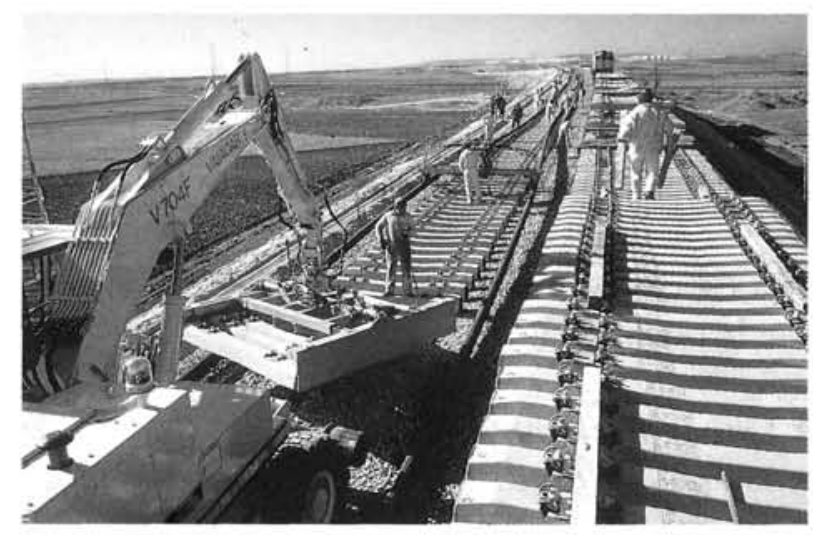

Descarga de traviesa en vía paralela.

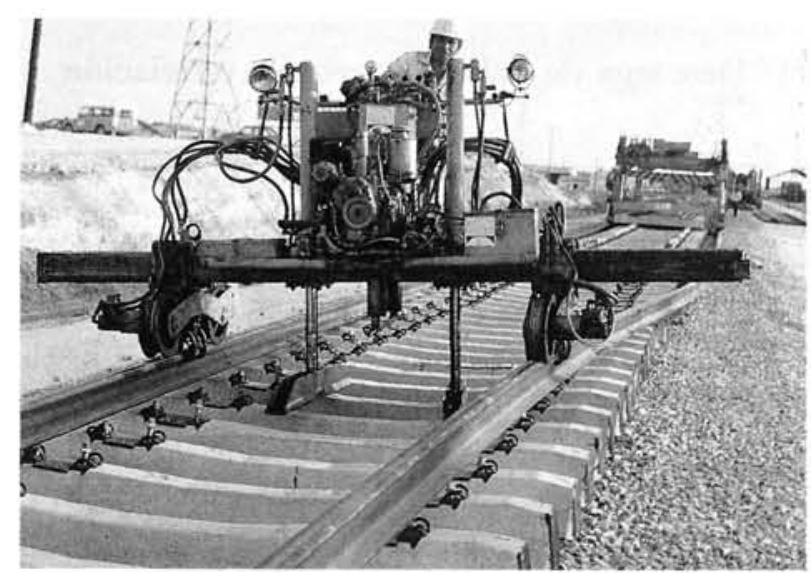

Posicionado de carril sobre traviesas. http://informesdelaconstruccion.revistas.csic.es 
Los carriles se posicionan de forma que las juntas queden centradas entre dos traviesas, con el fin de poder proceder a la soldadura de barras sin tener que mover las traviesas.

Una vez colocados los carriles se comprueba la posición de las traviesas, tanto en separación como en escuadría. La distancia entre traviesas de $60 \mathrm{~cm}$ debe respetarse con una tolerancia de $\pm 30 \mathrm{~mm}$. Asimismo, la distancia entre 6 traviesas consecutivas será de $3 \mathrm{~m} \pm 30 \mathrm{~mm}$.

Después de comprobada la posición de las traviesas se colocan los clips en posición de montaje, y con máquinas clavadoras, dotadas siempre con regulador de par de apriete, se procede a su apretado.

A continuación se comprueba el ancho de vía, debiendo corregir los defectos que se produzcan, fundamentalmente por falta de escuadría de las traviesas.

Para la circulación de los trenes de trabajo y maquinaria de vía, los carriles se embridan con bridas de emergencia (tipo C) que no necesitan taladro en los mismos.

Una vez clavada la barra larga soldada de $288 \mathrm{~m}$ se repite la operación de:

- Levante tramo vía auxiliar.

- Traslado al frente.

- Tendido vía auxiliar.

- Desplazamiento de los carriles auxiliares para pórticos hasta la siguiente BLS.

- Perfilado y cajeado del balasto.

- Descargar traviesas.

- Posicionar BLS.

- Clavar BLS.

\section{h) Descarga de balasto y primera nivelación}

Terminada la colocación de la vía en la forma descrita en las fases anteriores se descarga balasto mediante trenes de transporte formados por vagones-tolva de la serie 270.000 , con una capacidad de $36 \mathrm{~m}^{3}$ por tolva. A continuación se pasa la perfiladora para que se distribuya y regularice el balasto descargado.

Se procura arropar con balasto la vía recién clavada lo más pronto posible, sobre todo en épocas de gradiente térmico alto, para evitar que se produzcan deformacio- nes en sentido longitudinal que producen "garrotes» difíciles de corregir.

Previamente a los trabajos de $1 .{ }^{\text {a }}$ nivelación propiamente dichos se realizan trabajos de bateo, nivelaciones previas y alineación que, por medio de levantes y alineaciones sucesivas (máximo levante en cada pasada de $60 \mathrm{~mm}$ ), aproximan la vía a su posición definitiva en planta y alzado. El número de nivelaciones previas está en función del balasto colocado en 1 . capa.

Finalmente se realiza la llamada $1{ }^{\text {a }}$ nivelación, que son los trabajos necesarios para lograr que la vía se encuentre en su posición en planta, su cota esté entre -10 y $-20 \mathrm{~mm}$ de su cota teórica y todavía no se han liberado tensiones.

\section{i) Soldadura, liberación de tensiones y primera estabilización}

La siguiente operación es efectuar la soldadura aluminotérmica del carril y la liberación de tensiones. En esta operación se realiza el apretado de la clavazón, de la sujeción elástica $\mathrm{Hm}$, con un par de apriete de $210 \mathrm{~N}$.

A continuación se pasa la Estabilizadora Dinámica para realizar una primera estabilización.

Como normas generales hay que tener en cuenta lo siguiente:

- La teoría de la BLS está basada en que no se produzca deslizamiento del carril sobre las traviesas, salvo en su extremo, y esto se garantiza manteniendo constantemente un apretado correcto de la sujeción que, necesariamente, tiene que ser elástica para que su apretado sea constante.

- El anclaje de las traviesas al balasto, tanto longitudinal como transversalmente, es el elemento de seguridad más importante (supuesto el apretado de la clavazón) para la BLS. Para lograr esto el perfil de balasto debe estar completo y compactado, tanto las banquetas como los cajones.

- Siempre que haya en la vía algún elemento que no admita las tensiones, que por variación de temperatura pueden producirse, es necesario protegerle con aparato de dilatación. 
- Cualquier trabajo en la vía, que suponga una elevación o un ripado, aunque sea poco importante, lleva consigo una desconsolidación que no se recupera más que por una compactación del balasto con estabilizadora dinámica o por el paso de los trenes.

Durante la colocación de la vía es imposible conseguir que todas las traviesas de una BLS estén apretadas a una misma temperatura, no sólo por las variaciones de unos días a otros, sino por las que se producen en las horas que dura el trabajo diario.

Como consecuencia, cuando varía la temperatura, los efectos sobre una barra larga son diferentes en cada punto, ya que las tensiones son proporcionales a los incrementos de temperatura y éstos distintos, por haberlo sido los de apriete. Esto puede dar lugar a tres fenómenos perjudiciales:

- Rotura de carril por tracción (se ha montado con mucha temperatura y ésta desciende mucho).

- Pandeo por compresión (caso inverso al anterior).

- Pequeños movimientos laterales que producen un serpenteo en el carril que, en ocasiones, puede apreciarse a simple vista.

Para evitar estos problemas, se realiza la operación denominada "Liberación de tensiones", cuyo objetivo es conseguir que todos los puntos de una BLS sean fijados a la misma temperatura, logrando así que sus tensiones sean uniformes a cualquier temperatura.

Para lograrlo hay que determinar, en primer lugar, la temperatura de liberación, que se define como la media aritmética de las temperaturas máxima y mínima que alcancen los carriles durante el año, aumentada en $5^{\circ} \mathrm{C}$, admitiéndose una tolerancia de $\pm 3^{\circ} \mathrm{C}$.

Una vez determinada la temperatura de liberación y suponiendo que el carril está a una temperatura inferior a ella, lo cual es necesario siempre que se realiza esta operación, se puede actuar por dos procedimientos:

- Por calentamiento del carril (natural o artificial).

- Por tracción del carril.
El procedimiento normalmente utilizado es el de tracción del carril, toda vez que es más sencillo, preciso y está sujeto a menos errores humanos que el de calentamiento.

Las operaciones a realizar son las mismas, variando sólo el sistema de alargamiento del carril.

El fundamento de la liberación de tensiones es el siguiente:

Si tenemos un carril de cualquier longitud desclavado y suelto, a una temperatura (To), inferior siempre a la de liberación (Tl), y lo sometemos a la tensión que correspondería a una elevación de temperatura $(\mathrm{Tl}-\mathrm{To}$ ) con el carril inmovilizado y lo clavamos, cuando el carril alcance la temperatura $\mathrm{Tl}$ no tendrá ninguna tensión interna, es decir, se habrá conseguido el mismo efecto que si la clavazón hubiera sido apretada con el carril suelto a la temperatura de liberación.

La secuencia de la operación de liberación de tensiones es:

- Elección de la longitud a liberar.

- Constitución de puntos fijos.

- Aflojado de la clavazón y libre dilatación del carril.

- Medida de la temperatura de partida.

- Con la diferencia entre temperatura de liberación y la de partida, cálculo y marcaje de la variación de longitud del carril.

Para controlar que éste se ha dilatado en cada punto lo necesario, se hacen marcas en el patín del carril, cada $50 \mathrm{~m}$ refiriéndolo a las traviesas, con los desplazamientos que debe sufrir cada marca en función de su distancia al origen.

Asimismo, se establece la cala central entre los dos carriles a dilatarse. Esta cala es la correspondiente al incremento de longitud de ambos $(\mathrm{L} 1+\mathrm{L} 2)$ en función de la diferencia de temperatura $(\mathrm{Tl}-\mathrm{Tp})$ más 16-20 mm para luego hacer la soldadura en condiciones normales. 
El corte de carril necesario se hace con disco abrasivo, nunca con soplete.

- Alargamiento artificial del carril, por medio de tensores o calentamiento, hasta que coinciden las marcas establecidas.

- Fijación definitiva del carril y soldadura de cierre.

El apretado se realiza desde la soldadura hacia los puntos fijos:

- Retirada de los tensores. Se retiran una vez transcurridos un mínimo de 20 minutos, después de realizada la soldadura y terminado el apretado de la clavazón en, por lo menos, $30 \mathrm{~m}$ a cada lado de la soldadura.

- Homogeneización de tensiones en puntos fijos. Tiene por objeto conseguir un reparto de tensiones uniforme a lo largo de una cierta longitud de barra.

Consiste en aflojar la clavazón en la longitud del punto fijo, golpear el carril con mazos de madera, cobre o plástico (nunca metálico) y después apretar la sujeción lo más rápidamente posible para que se mantenga constante la temperatura.

Por último, cabe señalar que con temperaturas por debajo de $3^{\circ} \mathrm{C}$ no se debe realizar ninguna soldadura y, por tanto, no se puede efectuar la liberación de tensiones.

\section{j) Descarga de balasto, segunda nivelación y segunda estabilización dinámica}

Se realiza una nueva descarga de balasto con el tren de tolvas, y se pasa la perfiladora.

A continuación se realiza la segunda nivelación y una nueva estabilización dinámica.

Se dice que una vía está en 2. nivelación cuando se cumplen las condiciones siguientes:

- Ha estado en 1.a nivelación.

- Se han liberado tensiones.
- Se ha procedido a la 2a estabilización dinámica.

- La vía se encuentra en su posición correcta tanto en planta como en alzado.

- Se ha completado la banqueta de balasto, estando perfilado.

La estabilización dinámica pretende conseguir la compactación del balasto, bajo y alrededor de las traviesas, de forma artificial, para evitar tener que establecer limitaciones en la velocidad de circulación en la puesta en servicio de la línea.

Los estudios y ensayos realizados sobre el empleo del estabilizador dinámico en líneas de nueva construcción han puesto de manifiesto que, con su utilización, se logra un elevado efecto de compactación en el balasto situado bajo y alrededor de las traviesas sin que tenga influencia apreciable en las zonas colindantes. La compactación lograda es del orden de la que se consigue con el paso de 75.000 a 100.000 toneladas brutas.

La máquina estabilizadora se puede emplear de dos formas distintas:

- De ordinario se prefija el asiento deseado y un sistema automático regula la carga vertical para que, actuando en combinación con la frecuencia, se consiga el asentamiento previsto.

- En otros casos se desconecta el sistema automático y se usa la máquina con la máxima fuerza vertical y una frecuencia determinada. Cuando esto sucede el asentamiento no suele ser uniforme.

La forma de trabajo es la siguiente:

- Se emplea una frecuencia de $30 \mathrm{~Hz}$ a $35 \mathrm{~Hz}$ y una velocidad de trabajo entre $\operatorname{los} 1.000 \mathrm{~m} / \mathrm{h}$ y los 1.500 $\mathrm{m} / \mathrm{h}$.

- Después de cada levante se da una pasada del estabilizador, actuando con el regulador automático desconectado y una carga permanente del orden de 60 $\mathrm{kN}$ a $100 \mathrm{kN}$ por carril.

La cuantía de cada uno de estos levantes, medida después del bateo y antes de aplicar el estabilizador, debe estar comprendida entre $40 \mathrm{~mm}$ y $70 \mathrm{~mm}$. 
- Finalmente, se hace una última nivelación y después de ella una nueva pasada de estabilizador, pero actuando en este caso con el dispositivo automático de regulación de carga conectado.

En esta pasada de estabilizador se debe conseguir un descenso medio uniforme del orden de 5 a $10 \mathrm{~mm}$ utilizando la frecuencia y velocidad de avance citadas anteriormente. Los resultados obtenidos garantizan una resistencia lateral de $7,7 \mathrm{kN}$ para $2 \mathrm{~mm}$ de desplazamiento por traviesa. Esta resistencia aumenta con el paso sucesivo de las circulaciones.

En los túneles, viaductos y transición de plataforma a hormigón, se debe establecer una transición en la presión de trabajo, bajando ésta al $50 \%$ en los $30 \mathrm{~m}$ anteriores al punto singular. Una vez finalizada la obra o punto singular, en los $30 \mathrm{~m}$ siguientes se vuelve a establecer una transición para seguir estabilizando al 100\%.

Simultáneamente, aprovechando los trenes necesarios para el transporte de materiales al tajo de avance, se hace transitar por el trayecto estabilizado un tráfico del orden de 75.000 a 100.000 t brutas, lo que contribuye eficazmente el aumento de la resistencia lateral. La experiencia obtenida garantiza, por este hecho, un aumento de la resistencia lateral del orden del 10 al $20 \%$ sobre la obtenida con el simple empleo del estabilizador dinámico, pudiéndose abrir al tráfico la nueva vía sin limitación alguna de velocidad respecto a la proyectada.

En el gráfico de las págs. siguientes se reflejan las operaciones antes descritas, así como las secuencias de las mismas.

\section{k) Amolado de carril}

Previo a la puesta en servicio comercial de una línea de alta velocidad, se ha demostrado que es imprescindible el amolado de carril con el fin de facilitar el mantenimiento de la vía y los aparatos, mejorar las condiciones que permiten altas velocidades de circulación y reducir el ruido producido por la interacción rueda-carril que, a estas velocidades, es el predominante.

Con este amolado preventivo se logra:

- Eliminar las ondas largas (de 25 a $225 \mathrm{~cm}$ de longitud) resultantes de la laminación del carril. (c) Consejo Superior de Investigaciones Científicas Licencia Creative Commons 3.0 España (by-nc)

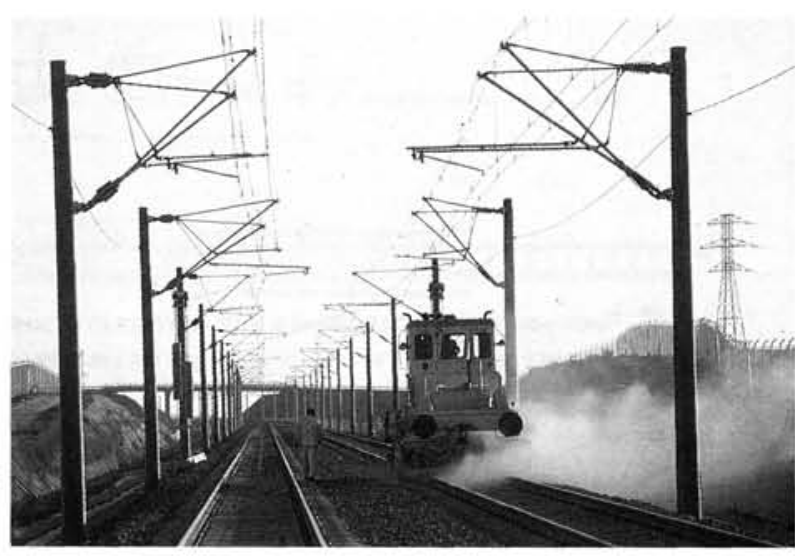

Trabajos de perfilado de vía.

- Eliminar los defectos puntuales de carril.

- Evitar que los ligeros defectos del carril den lugar a otros más importantes.

- Retrasar la posible aparición del desgaste ondulatorio.

- Lograr una rugosidad de carril compatible con las velocidades a desarrollar.

El tren después de obtener los gráficos de la situación inicial, efectúa el trabajo de amolado en tres fases.

La primera fase se efectúa con las unidades bloqueadas, con el fin de eliminar las ondas largas y parte de las pequeñas.

En la segunda fase, y ya con las unidades libres, se eliminan las ondas cortas que pueden dar lugar al desgaste ondulatorio.

Finalmente, se reperfila el carril para aproximarlo a su perfil teórico. Las tolerancias que se admiten son de $\pm 0,3 \mathrm{~mm}$ iguales a las de fabricación del carril. 

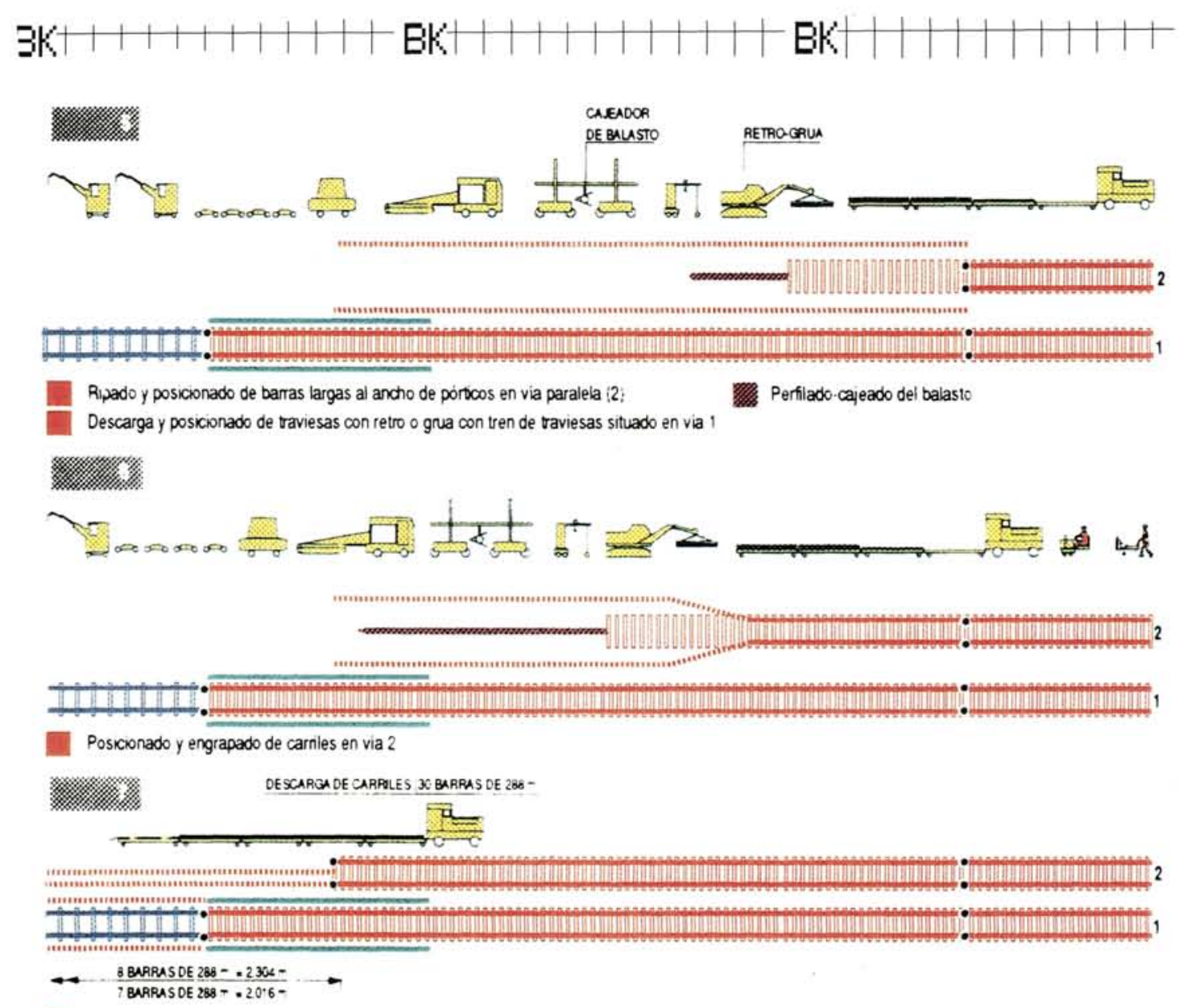

Se itera el proceso con la descarga de un nuevo tren camiero

IREN DE BAUSTO

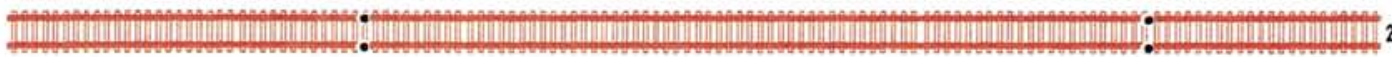

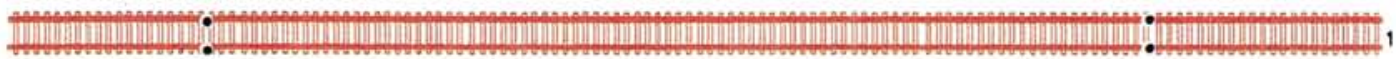

Descarga del tren de balasto. perfilado de regularización y distrbucion

Alineación, nivelación-bateo por levantes sucesivos hasta 14 nivelacion y perfilado
Soldadura aluminotermica y liberacion de tensiones

14 Estabilizacion dinámica

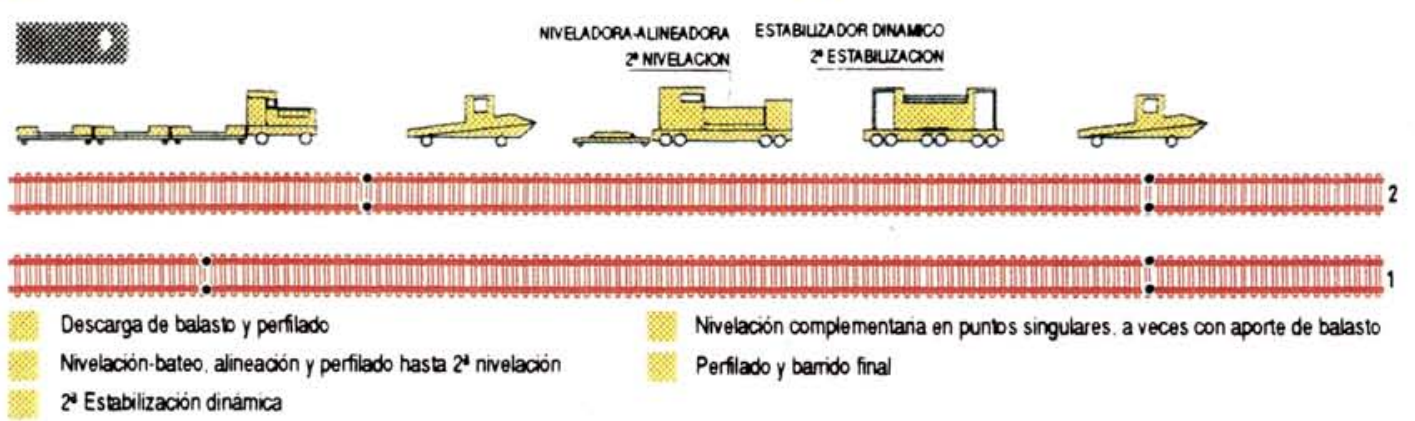


Organización del montaje de vía.

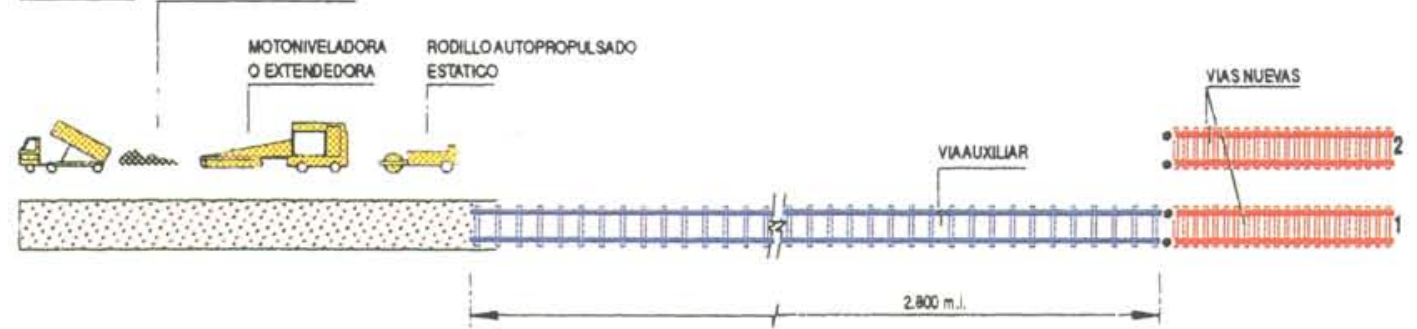

2. Descarga y extendido 1 capa de balasto con motoniveladora o extendedora

Colocación de via auxiliar ancho internacional con carril usado y traviesas de madera

\section{.}

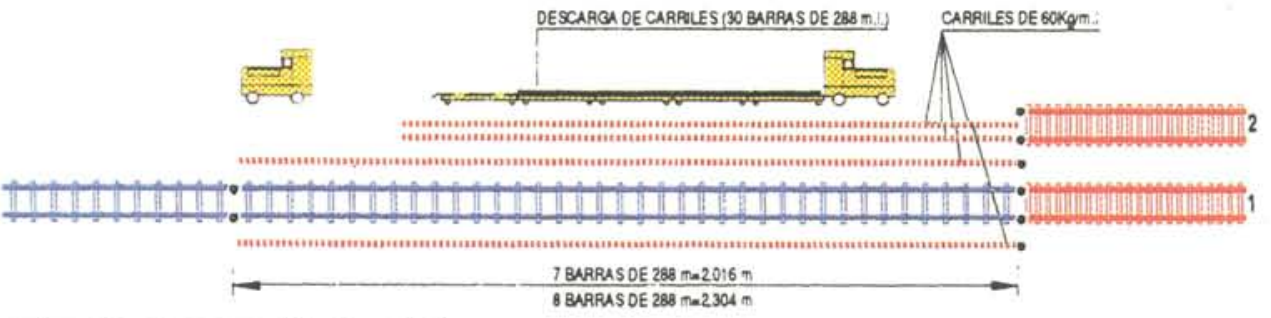

Descarga de ten carrilero 14 barras en via 1 y 16 en via 20 viceversa

Embridado y posicionado de barras largas al ancho porticos en via 1

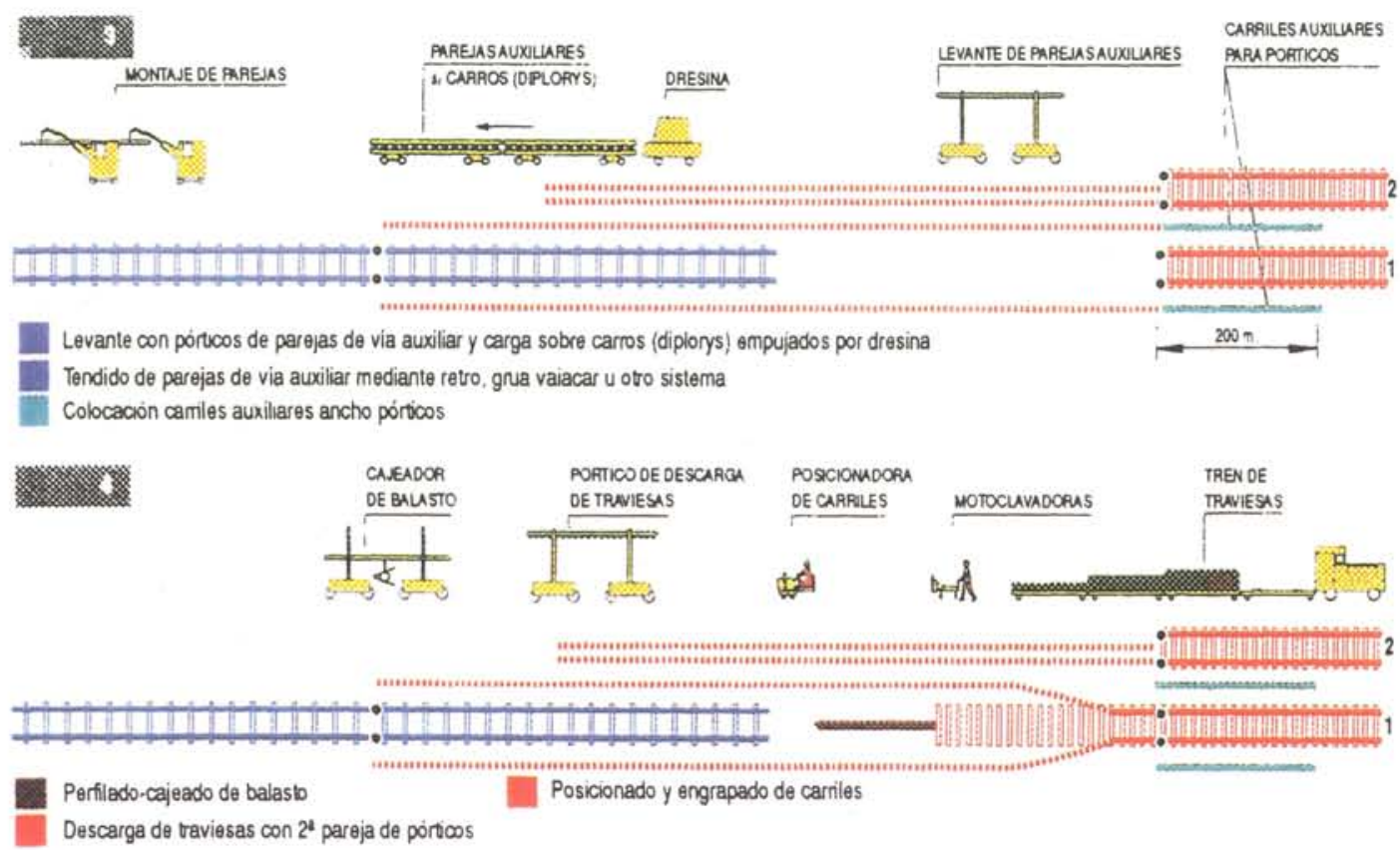




\section{LA RECEPCIÓN DE LA VÍA}

La recepción de la vía se realiza cuando los trabajos están efectuados de modo técnicamente correcto sin defectos esenciales.

Para poder recepcionar una vía es preciso establecer unos valores límites de tolerancias en cada uno de los parámetros que definen la calidad de una vía, así como cumplirse una serie de condiciones.

Para proceder a la recepción de la vía se dispondrá de:
- Los registros del vehículo de auscultación geométrica con análisis de los mismos.

- Registros de control de las máquinas bateadorasalineadoras y del estabilizador dinámico de vía.

- Actas de comprobación del estado de la vía y en las que se recojan los parámetros que lo definen.

- Hojas kilométricas y Actas de liberación de tensiones.

Aunque todas las diferencias de los datos teóricos y reales deben tender a cero, las tolerancias límite establecidas para una recepción definitiva de vía aparecen en el cuadro 1 .

\section{CUADRO 1 \\ VALORES LÍMITE PARA LA RECEPCIÓN DE LA VÍA}

\begin{tabular}{|c|c|c|}
\hline MAGNITUD MEDIDA & VALOR LÍMITE & OBSERVACIONES \\
\hline Nivelación Longitudinal & $+10 \mathrm{~mm} /-20 \mathrm{~mm}$ & $\begin{array}{l}\text { Desviaciones de la posición teórica en la línea de postes de cate- } \\
\text { naria enfrentados. }\end{array}$ \\
\hline & $\begin{array}{l}10 \mathrm{~mm} \\
3 \mathrm{~mm}\end{array}$ & $\begin{array}{l}\text { Diferencia, en valor absoluto, entre postes vecinos. } \\
\text { Diferencia cada } 5 \mathrm{~m} \text {, en valor absoluto. }\end{array}$ \\
\hline Alineación & $\pm 10 \mathrm{~mm}$ & $\begin{array}{l}\text { Desviaciones de la posición teórica, en valor absoluto, en línea de } \\
\text { postes de catenaria enfrentados. }\end{array}$ \\
\hline & $3 \mathrm{~mm}$ & $\begin{array}{l}\text { Diferencia cada } 5 \mathrm{~m} \text {, en valor absoluto, medida con cuerda de } 20 \\
\text { m avanzando de } 5 \text { en } 5 \mathrm{~m} \text {. }\end{array}$ \\
\hline Peralte & $\pm 2 \mathrm{~mm}$ & Medida cada $5 \mathrm{~m}$. \\
\hline Ancho de vía & $-3 \mathrm{~mm}$ & $\begin{array}{l}\text { Se comprobará la posición a escuadra cuando el ancho sea infe- } \\
\text { rior a } 1.435 \mathrm{~mm} \text {. }\end{array}$ \\
\hline Apriete de sujeción & $-20 \mathrm{Nm} /+30 \mathrm{Nm}$ & $\begin{array}{l}\text { Se comprobará que la parte central del bucle apoya en la placa } \\
\text { sobre } 230 \mathrm{Nm} \text {. }\end{array}$ \\
\hline Distribución de traviesas & $\begin{array}{l} \pm 30 \mathrm{~mm} \text { aisladamente }+50 \\
\mathrm{~mm} \text { excepcionalmente }\end{array}$ & $\begin{array}{l}\text { En } 36 \mathrm{~m} \text { se comprobará que hay } 60 \text { traviesas, con tolerancia de } \\
\quad \pm 30 \mathrm{~mm} \text {. }\end{array}$ \\
\hline Soldaduras & $\begin{array}{l}+0,3 \mathrm{~mm} /-0,2 \mathrm{~mm} \text {. Superfi- } \\
\text { cie de soldadura } \\
\text { Borde activo: } 0,3 \mathrm{~mm} \text { hacia } \\
\text { afuera, } 0 \mathrm{~mm} \text { hacia dentro. }\end{array}$ & Medida con regla de 1 metro de longitud. \\
\hline
\end{tabular}


El estado de una vía se mide, tanto por su situación estática, como por su comportamiento dinámico. Aunque en éste intervienen varios condicionantes, el más importante es el cumplimiento de los parámetros geométricos que califican la vía:

\section{a) Auscultación de vía}

Al estar dotada la nueva línea de Alta Velocidad de ancho internacional, no era posible realizar la auscultación con el coche de control geométrico de que dispone REN$\mathrm{FE}$, al ser bastante compleja la modificación de los sistemas de medición de éste y no disponer de un programa de análisis que mida de acuerdo con las tolerancias de vía fijadas y que son distintas a las utilizadas habitualmente por RENFE.

$\mathrm{Al}$ ser las tolerancias las mismas que tienen los ferrocarriles alemanes para sus líneas de Alta Velocidad se ha contratado el empleo del vagón auscultador que ha servido para la auscultación de aquellas líneas.

El método de medición utilizado proporciona, tanto gráfica como numéricamente, los parámetros que definen la calidad de la vía.

Los valores de medición determinados con el tren medidor se procesan según los métodos de valoración del cuadro 2.

En el análisis, los valores individuales se comparan con los límites que se fijan y se clasifican por clases.

Los defectos de Clase 1 son aquellos que están dentro de tolerancias.

Los de Clase 2, son defectos pequeños. El ordenador los escribe en el punto kilométrico (P.K.) correspondiente, pero no los contabiliza en el resumen de defectos.

Los de Clase 3 (defectos medianos) y Clase 4 (defectos grandes) los escribe en su KM correspondiente y los contabiliza en los resúmenes de los tramos en $\mathrm{Kl}_{3}$ y $\mathrm{Kl}_{4}$.

Los valores límites de calidad se recogen en el cuadro 3 , pudiéndose proceder a la recepción de la vía cuando se cumplen todos los valores.
CUADRO 2

\begin{tabular}{|l|l|}
\hline \multicolumn{1}{|c|}{ PARÁMETROS } & \multicolumn{1}{|c|}{ MÉTODO DE VALORACIÓN } \\
\hline $\begin{array}{l}\text { Nivelación lon- } \\
\text { gitudinal }\end{array}$ & De pico a pico \\
Alabeo & $\begin{array}{c}\text { De valor medio al pico y del 0 al } \\
\text { pico }\end{array}$ \\
Ancho de vía & De $1.435 \mathrm{~mm}$ al pico \\
Peralte & De valor medio al pico \\
Alineación & De pico a pico \\
\hline
\end{tabular}

El significado de estos métodos de valoración es el siguiente:

"De pico a pico»: Ajustar entre valores máximos y mínimos sucesivos.

"De valor medio a picon: Distancias de los valores extremos desde el valor medio, que se forma en una longitud de $20 \mathrm{~m}$.

«De $1.435 \mathrm{~mm}$ al picon: Distancia de los valores extremos desde el valor de $1.435 \mathrm{~mm}$.

"Del 0 al pico»: Distancia de los valores extremos desde 0.

\section{b) Pruebas de comportamiento dinámico}

Una vez finalizada la implantación de la vía en la nueva línea, es preciso evaluar el comportamiento del sistema vehículo-vía mediante ensayos de circulación real.

Estas circulaciones experimentales realizadas por el Laboratorio Central de RENFE en locomotoras, coche laboratorio y Rama AVE, permiten estudiar la calidad dinámica de la marcha de los vehículos y la respuesta de la vía al paso de estas circulaciones.

Las medias que se realizan son para determinar:

- Las acciones sobre la vía (estabilidad, confort y calidad de marcha) así como su control.

- Determinación de defectos. 
CUADRO 3

VALORES LÍMITE DE CALIDAD

\begin{tabular}{|c|c|c|c|c|c|c|}
\hline \multirow{3}{*}{$\begin{array}{l}\text { No de código } \\
\text { del programa } \\
\text { de evaluación }\end{array}$} & \multirow{3}{*}{$\begin{array}{l}\text { Campo de } \\
\text { aplicación }\end{array}$} & \multicolumn{5}{|c|}{$\begin{array}{l}\text { Valores característicos de los parámetros geométricos } \\
\text { de la vía con las correspondientes clases de defectos }\end{array}$} \\
\hline & & $\begin{array}{l}\text { Nivelación } \\
\text { longitudinal } \\
\text { (mm) }\end{array}$ & $\begin{array}{c}\text { Alabeo } \\
(\%) \\
\text { de acuerdo con }\end{array}$ & $\begin{array}{c}\text { Ancho de } \\
\text { Vía } \\
(\mathrm{mm})\end{array}$ & $\begin{array}{c}\text { Nivelación } \\
\text { transversal } \\
\quad(\mathrm{mm}) \\
\text { de evaluación }\end{array}$ & $\begin{array}{l}\text { Alineación } \\
\text { (mm) }\end{array}$ \\
\hline & & $\begin{array}{l}\text { Puntal } \\
\text { Punta }\end{array}$ & $\begin{array}{l}\text { Valor medio/ } \\
\text { Punta }\end{array}$ & $\begin{array}{l}1.435 / \\
\text { Punta }\end{array}$ & $\begin{array}{l}\text { Valor medio/ } \\
\text { Punta }\end{array}$ & $\begin{array}{l}\text { Puntal } \\
\text { Punta }\end{array}$ \\
\hline $6.011 / 0$ & $\begin{array}{l}\text { Vías con } \\
\text { materiales } \\
\text { nuevos }\end{array}$ & 6 & 1,3 & $-3 /+3$ & 4 & 6 \\
\hline
\end{tabular}

\section{Acciones sobre la vía. (Estabilidad y seguridad en la rodadura)}

Fundamentalmente son los esfuerzos laterales y verticales $(\mathrm{Y}, \mathrm{Q})$ transmitidos por el eje del vehículo a la vía en los puntos de contacto rueda-carril.

De la cuantía de estos esfuerzos y de su relación Y/Q depende, principalmente, la seguridad de rodadura de un vehículo ferroviario, ya que de su conjunción dependen a su vez:

- el equilibrio del eje (riesgo de descarrilamiento),

- el equilibrio de la vía (riesgo de ripado).

El esfuerzo lateral límite para que no exista riesgo de ripado de la vía, según el criterio de Proud'home, que está admitido internacionalmente por todas las Administraciones ferroviarias del ámbito de la UIC, viene dado por el valor de:

$$
H=1+P / 3 \text {, expresado en } t
$$

donde :

$\mathrm{H}=$ Esfuerzo transversal total eje-vía.

$\mathrm{p}=$ Peso del eje.
El valor admisible de esfuerzo $\mathrm{H}$ para explotación ferroviaria se fija en el $85 \%$ del valor límite citado mantenido en $2 \mathrm{~m}$ de vía.

$$
\mathrm{H}_{(2 \mathrm{~m})}=0,85(1+\mathrm{P} / 3)
$$

Con la relación de esfuerzos laterales y verticales, a nivel de rueda, se establece el coeficiente de descarrilamiento $(\mathrm{Y}, \mathrm{Q})$, y su valor máximo admisible depende del perfil de rodadura y del ángulo de pestaña, aunque se recomienda $\mathrm{Y} / \mathrm{Q} \leq \mathbf{0 , 8}$ (mantenido en $2 \mathrm{~m}$ ), en ciertos casos se admiten valores superiores en función de la conicidad equivalente.

\section{Medida de los Esfuerzos Transversales y Vertica-} les.-Se pueden medir por dos métodos:

\section{Método directo}

Ambos esfuerzos se pueden medir directamente a nivel de contacto rueda-carril mediante ejes dinamométricos especialmente instrumentados.

\section{Método indirecto}

Cuando no existen ejes dinamométricos: 


\section{- Esfuerzos Transversales}

Dado que la fuerza lateral transmitida por un eje a la vía es debida principalmente a los esfuerzos de inercia originados por los movimientos de lazo de caja y bogie (ya que en un vehículo totalmente estable los esfuerzos debidos a la rodadura son mínimos), se pueden conocer los esfuerzos $\mathrm{H}$ mediante la medida de aceleraciones transversales en caja y bogie en puntos concretos, según la geometría del vehículo y estableciendo una relación entre ellas, en función de las masas que soportan mediante el cálculo de momentos de inercia respecto a los ejes donde queremos medir los Esfuerzos $\mathrm{H}$.

$\mathrm{H}_{1}=\mathrm{m}_{1} A b_{1}+\mathrm{m}_{2} A b_{2}+\mathrm{m}_{\mathrm{c}} \mathrm{Ac}$

$\mathrm{H}_{2}=\mathrm{m}_{2} \mathrm{Ab} \mathrm{b}_{1}+\mathrm{m}_{1} \mathrm{Ab}_{2}+\mathrm{m}_{\mathrm{c}} \mathrm{Ac}$

En un vehículo simétrico

donde:

$\mathrm{H}_{1}$ y $\mathrm{H}_{2}=$ son los dos ejes de un mismo bogie.

$A b_{1}$ y $A b_{2}=$ Aceleraciones transversales en bogie.

$\mathrm{Ac}=$ Aceleración en caja del vehículo.

$\mathrm{m}_{1}, \mathrm{~m}_{2}$ y $\mathrm{m}_{\mathrm{c}}=$ Coeficientes proporcionales a las masas soportadas.

Este método indirecto para esfuerzos transversales da una buena aproximación de los esfuerzos reales para trazados rectos o de curvas de radio grande; no así para trazados de curvas de radio reducido donde hay contactos continuos de pestaña-carril que invalidan las premisas teóricas que lo sustentan.

\section{- Esfuerzos Verticales}

Estos esfuerzos son debidos a la variación de carga vertical y están provocados principalmente por los movimientos verticales y de balanceo a nivel de caja y por los defectos de vía a nivel de masas no suspendidas.

Se pueden medir indirectamente midiendo el desplazamiento de los amortiguadores de la suspensión primaria, teniendo en cuenta su constante de elasticidad, el radio de rodadura, el empate de bogie, el ancho de vía y la situación de los captadores que miden el desplazamiento, además de los esfuerzos $\mathrm{H}$.

$$
\begin{array}{ll}
\triangle \mathrm{Q}_{1}=\mathrm{K}\left(\mathrm{aD}_{1}-\mathrm{bD}_{2}\right)+\mathrm{cH} & \mathrm{Q}_{1}=\mathrm{Q}_{0}+\triangle \mathrm{Q}_{1} \\
\triangle \mathrm{Q}_{2}=\mathrm{K}\left(\mathrm{aD}_{2}-\mathrm{bD}_{1}\right)-\mathrm{cH} & \mathrm{Q}_{2}=\mathrm{Q}_{0}+\triangle \mathrm{Q}_{2}
\end{array}
$$

donde:

$\triangle \mathrm{Q}_{1}$ y $\triangle \mathrm{Q}_{2}=$ Variaciones dinámicas de la carga vertical sobre ruedas.

$Q_{1}$ y $Q_{2}=$ Esfuerzos verticales totales sobre las ruedas. $\mathrm{Q}_{0}=$ Carga estática por rueda.

$D_{1}$ y $D_{2}=$ Desplazamientos de la suspensión primaria. $\mathrm{K}=$ Constante de elasticidad de los amortiguadores.

$a, b$ y $c=$ Coeficientes.

Complementariamente y en relación con la estabilidad se miden aceleraciones transversales en bogie, y desplazamientos laterales y verticales caja-bogie, cuya situación y valoración depende de cada caso. Analizándose tanto valores sostenidos como máximos y eficaces.

\section{Confort y Calidad de marcha}

Se determinan mediante la medida de aceleraciones transversales y verticales en caja del vehículo. Aunque dichas medidas son referidas al vehículo de que se trate, la influencia de la calidad de vía sobre el mismo es notoria.

La nota de Confort permite la apreciación del vehículo desde el punto de vista de la persona que viaja en él, y se evalúa según la norma ISO 2631 (modificada para el transporte ferroviario), que la denomina Límite a la capacidad reducida por fatiga y se da en horas.

Esta nota se obtiene mediante el cálculo de la aceleración eficaz ponderado (según la norma) de las aceleraciones, y su interpolación lineal entre los puntos que figuran en dicha norma.

El índice de calidad de marcha o índice de Sperling califica al vehículo ferroviario desde el punto de vista de la calidad de marcha (respuesta del vehículo a las solicitaciones de la vía) y se establece de acuerdo con la siguiente tabla:

\begin{tabular}{|c|l|}
\hline Wz & \multicolumn{1}{|c|}{ Calidad de Marcha } \\
\hline 1 & Muy buena. \\
2 & Buena. \\
3 & Satisfactoria. \\
4 & Todavía apto para el servicio. \\
5 & Peligroso para la explotación. \\
\hline
\end{tabular}

El límite establecido por la UIC para la admisibilidad de un vehículo es: $\mathrm{Wz} \leq 4,25$. 
Asimismo, se establece otro índice en relación con la calidad de marcha, que es la Aceleración ponderada (Sp), que se da en unidades de $\mathrm{m} / \mathrm{s}^{2}$ y se aplica a todo tipo de aceleraciones medidas tanto en caja como en bogie, y su valor máximo admisible es: $\mathrm{Sp} \leq 1,3 \mathrm{~m} / \mathrm{s}^{2}$.

Ambos índices se calculan por funciones de ponderación establecidas en la ficha UIC 432-OR.

\section{Control de calidad de vía mediante la medida de aceleraciones en rama AVE}

Con estas medidas se detectan:

- Defectos puntuales laterales.-Dos aceleraciones transversales en bogie.

- Defectos puntuales verticales.-Una aceleración vertical en caja de grasa de un eje.

\section{- Defectos no puntuales, o de mayor duración.-} Aceleraciones vertical y transversal en caja de vehículo.

Los defectos laterales puntuales se reflejan claramente en cualquier punto del bastidor del bogie. Dado que el propio vehículo puede generar movimientos análogos, éstos se discriminan realizando la misma medida en dos bogies. distintos próximos.

Los defectos verticales puntuales, por su propia naturaleza, acaban apareciendo en ambos carriles, y son fácilmente detectables con una sola medida en una caja de grasa de un eje.

Con la medida de aceleraciones en caja del vehículo se detectan, a través de la suspensión secundaria, los defectos no puntuales o de mayor duración que no siempre se reflejan en las medidas anteriores. Asimismo, también aparecen los defectos puntuales, pero atenuados o distorsionados y a veces de difícil identificación, que siempre se pueden discriminar gon las medidas en bogie y caja de grasa.

Además de las aceleraciones, también se miden la velocidad y el punto kilométrico del tren, para determinar a qué velocidad se produjo el defecto y su situación en la línea. Estos parámetros se obtienen midiendo en fre- cuencia una señal procedente de un generador tacométrico de un eje de un bogie remolque.

Esta información, analizada sobre registro gráfico, califica el estado de la vía en:

1. Puntos donde se requiere la actuación inmediata de mantenimiento.

2. Puntos donde se requiere, asimismo, la actuación de mantenimiento, aunque no de forma inmediata.

3. Puntos que requieren seguimiento en la evolución del defecto detectado.

Los criterios de ponderación actualmente utilizados a partir de la medida son:

1. En la actuación inmediata:

- Aceleración lateral de bogie $\geq 6 \mathrm{~m} / \mathrm{s}^{2}$ (filtrada a $10 \mathrm{~Hz}$ ).

- Aceleración vertical en caja de grasa $\geq 60 \mathrm{~m} / \mathrm{s}^{2}$ (filtrada a $20 \mathrm{~Hz}$ ).

2. En actuación no inmediata:

- Aceleración lateral de bogie $\geq 4 \mathrm{~m} / \mathrm{s}^{2}$ (filtrada a $10 \mathrm{~Hz}$ ).

- Aceleración vertical en caja de grasa $\geq 40 \mathrm{~m} / \mathrm{s}^{2}$ (filtrada a $20 \mathrm{~Hz}$ ).

- Aceleraciones de caja con valores sostenidos de al menos 6 ciclos con niveles $\geq 1 \mathrm{~m} / \mathrm{s}^{2}$.

3. Para seguimiento:

- Aceleración lateral de bogie $\geq 3 \mathrm{~m} / \mathrm{s}^{2}(10 \mathrm{~Hz})$.

- Aceleración vertical en caja de grasa $\geq 30 \mathrm{~m} / \mathrm{s}^{2}$ $(20 \mathrm{~Hz})$.

Estas medidas, no sólo se efectúan para evaluar la situación de la vía antes de su puesta en Explotación, sino que se siguen realizando periódicamente ( 2 al mes) para, de acuerdo con los resultados obtenidos, evaluar el estado de la línea y programar los niveles de actuación correspondientes. 